\title{
Are musculoskeletal conditions neglected in national health surveys?
}

\author{
Giovanni E. Ferreira ${ }^{1,2}$, Rachelle Buchbinder ${ }^{3,4}$, Joshua R. Zadro ${ }^{1,2}$, Mary \\ $\mathrm{O}^{\prime} \mathrm{Keeffe}^{2}$, Priti Kharel ${ }^{1,2}$, Lidia Carballo-Costa ${ }^{5}$, Juliana S. Oliveira ${ }^{1,2}$ and \\ Christopher G. Maher ${ }^{1,2}$
}

${ }^{1}$ Sydney School of Public Health, Faculty of Medicine and Health, University of Sydney, ${ }^{2}$ Institute for Musculoskeletal Health, The University of Sydney and Sydney Local Health District, Sydney, NSW, ${ }^{3}$ Monash Department of Clinical Epidemiology, Cabrini Institute, ${ }^{4}$ Department of Epidemiology and Preventive Medicine, School of Public Health \& Preventive Medicine, Monash University, Melbourne, VIC, Australia and ${ }^{5}$ Department of Physiotherapy, Medicine and Biomedical Sciences, Universidade da Coruña, Faculty of Physiotherapy, Coruña, Spain

\begin{abstract}
Objective. To describe the proportion of national health surveys that contain questions on the prevalence and consequences of musculoskeletal conditions.

Methods. We used a comprehensive search strategy to obtain national health surveys from the 218 countries listed by the World Bank. Two authors independently extracted information from each national health survey. Outcomes were the proportion of surveys that contained questions on the prevalence of musculoskeletal conditions using the Global Burden of Disease categorization of RA, OA, low back pain, neck pain, gout and other and contained condition-specific questions about activity limitation, severity of pain and work absence. We also measured how frequently the prevalence of low back pain was measured using a consensus-based standard definition for low back pain prevalence studies.

Results. We identified national health surveys from 170 countries. Sixty-two (36.4\%), the majority from highincome countries $(n=43)$, measured the prevalence of at least one musculoskeletal condition. OA [53 (85.4\%)], low back pain [39 (62.9\%)] and neck pain [37 (59.7\%)] were most commonly measured, while RA and gout prevalence were only measured in $10(5.9 \%)$ and $3(1.8 \%)$ surveys, respectively. A minority of surveys assessed condition-specific activity limitations [6 (3.6\%)], pain severity [5 (2.9\%)] and work absence [1 $(0.6 \%)]$. Only one survey used the consensus-based standard definition for low back pain.

Conclusion. Musculoskeletal conditions are neglected in the majority of national health surveys. Monitoring musculoskeletal conditions through ongoing surveys is crucial for the development and evaluation of health policies to reduce their burden.
\end{abstract}

Key words: low back pain, osteoarthritis, rheumatic diseases, health policy, public health 


\section{Introduction}

Musculoskeletal health conditions rank fifth in terms of disability-adjusted life years worldwide [1]. Among this group of conditions, low back pain is the biggest contributor to the disability pool, with a worldwide point prevalence of $7.8 \%$, affecting 577 million people at any one time [2]. Combined, low back and neck pain were responsible for the highest healthcare spending in the USA in 2016 among all diseases [3]. The burden of rheumatic diseases such as RA and gout is also increasing worldwide. For example, since 1990, the burden of RA has increased by 7.4\% [4]. It is unclear to what extent countries have established processes to regularly monitor progress in addressing the burden of musculoskeletal conditions [5]. Further, there is a lack of standardization of definitions used in prevalence studies [6] and many prevalence studies use definitions that rely only on the presence of pain, not on the impact of symptoms on activity limitation or work absence [7]. The lack of agreement on which definition to use is also a problem. For example, among the level 4 musculoskeletal conditions listed in the Global Burden of Disease Study, only low back pain appears to have an internationally agreed standardized definition to be used in prevalence studies [8]. Another problem is the lack of data from low- and middle-income countries (LMICs) that might provide an indication that the burden of musculoskeletal conditions is underestimated in these countries [5].

National health surveys are an important source of data for public health surveillance and health service planning. They usually collect data on a broad range of topics, such as access to health services, prevalence of disease and care-seeking behaviours [9]. National health surveys are considered the main data source to support evidence-based policy planning, development and monitoring $[10,11]$ and therefore represent an important tool to quantify the prevalence of musculoskeletal conditions. To the best of our knowledge, no previous study has systematically considered the way data about the prevalence of musculoskeletal conditions and their impact on activity limitation and work absence are collected in national health surveys.

The aim of this study was to describe the proportion of national health surveys that contained questions on the prevalence of musculoskeletal conditions and to which musculoskeletal conditions they referred and questions about activity limitation, severity of pain and work absence due to musculoskeletal conditions. We also determined whether any national health surveys had used the consensus-based definition for low back pain prevalence studies recommended by Dionne et al. in 2008 [12].

\section{Methods}

\section{Eligibility criteria}

We searched for the most recent national health surveys from the 218 countries listed by the World Bank. We defined a national health survey as a survey rolled out by a national government body, or by a third-party organization on behalf of the national government, intended to provide nationally representative estimates on a wide range of health status and utilization measures in the population. We excluded disease-specific surveys and surveys conducted at the city, county or state level. We did not place any restrictions on the language of the survey and attempted to translate documents in any language. 


\section{Search strategy}

We searched Google, the Global Health Data Exchange website (http://ghdx.healthdata.org/) and each country's National Statistics Office website. We conducted searches from 26 November to 10 December 2019. A detailed description of the search methods is presented in Supplemental File 1, available at Rheumatology online. Two authors conducted searches independently. Documents deemed relevant by both researchers were included. Disagreements were resolved by discussion.

\section{Data extraction and coding}

One reviewer extracted data and a second reviewer independently checked the data. Disagreements were resolved by consensus. Data were extracted preferentially from questionnaires or from the full report or executive summary. We extracted data on the income level of each country according to the 2019 World Bank classification (i.e. lower income, lower-middle income, upper-middle income, high income) and characteristics of the survey (e.g. geographical region, language, response rate, sample size, age range).

We extracted data from questions related to the six level 4 musculoskeletal conditions listed in the 2017 Global Burden of Disease Study: low back pain, neck pain, OA, RA, gout and other musculoskeletal [1]. We considered as 'other musculoskeletal' any condition that was clearly described and for which we could assign an International Classification of Diseases, Tenth Revision diagnostic code (e.g. fibromyalgia - M79.7). We extracted data on the anatomical site, prevalence period, symptom duration, pain severity, activity limitation and disease-related work absence. We also coded whether a question referred to 'acute' or 'chronic' symptoms and if a duration-specific definition was provided.

For low back pain, we coded whether questions used the minimal or optimal definitions of low back pain proposed by Dionne et al. [8]. For either definition to be satisfied, all items as described by the original study, with the exception of the body diagram, had to be present (Supplemental File 2, available at Rheumatology online). For OA, RA and gout, in addition to a self-reported diagnosis, we coded whether a survey had a question about self-reported doctor-diagnosed OA. We did not consider this question for low back and neck pain because they are symptoms rather than conditions and therefore it is appropriate that these are self-reported [2].

\section{Outcomes}

Outcomes were the proportion of national health surveys that contained questions on the prevalence of specific musculoskeletal conditions; contained condition-specific questions about activity limitation, severity of pain and work absence; and measured the prevalence of low back pain following standardized minimal or optimal definitions as proposed by Dionne et al. [8].

For national health surveys with rotating blocks of questions included in the questionnaire on some years with fixed periodicity (e.g. the US National Health Interview Survey), we considered the survey to have the questions if they were part of a rotating block of questions.

\section{Data analysis}

We presented data for each income category separately. Characteristics of the national health surveys were presented using counts and percentages or means and S.D.s where appropriate. Outcomes were presented as frequencies and proportions. 


\section{Results}

We retrieved national health surveys from 170 of 218 countries $(78 \%)$. The highest percentages of national health survey retrieval were from lower-middle-income (95.7\%) and low-income countries (93.6\%). The year of publication ranged from 1991 to 2020 . Almost $84 \%$ of the national health surveys were published in the last 10 years. Respondents $\geq 65$ years of age were not interviewed in almost half of the national health surveys (Table 1). Supplemental File 3, available at Rheumatology online, describes additional data on the retrieved surveys.

TABLE 1 Economic and geographical characteristics of countries with an identified national health survey $(\mathrm{N}=170)$

\begin{tabular}{lc}
\hline Variable & $n(\%)$ \\
\hline & \\
Income classification & $54(67.5)$ \\
$\quad$ High income $(n=80)^{\mathrm{a}, \mathrm{b}, \mathrm{c}}$ & $43(71.6)$ \\
Upper-middle income $(n=60)$ & $44(95.7)$ \\
Lower-middle income $(n=47)^{\mathrm{a}}$ & $29(93.6)$ \\
Low income $(n=31)$ & \\
Region & $30(78.9)$ \\
East Asia and Pacific $(n=38)^{\mathrm{a}}$ & $41(70.7)$ \\
Europe and Central Asia $(n=58)^{\mathrm{a}}$ & $26(61.9)$ \\
Latin America and Caribbean $(n=42)$ & $13(65.0)$ \\
Middle East and North Africa $(n=21)^{\mathrm{a}}$ & $3(100)$ \\
North America $(n=3)$ & $8(100)$ \\
South Asia $(n=8)$ & $46(95.8)$ \\
Sub-Saharan Africa $(n=48)$ & $85(50.0)$ \\
Published in the last 5 years & $142(83.5)$ \\
Published in the last 10 years & $80(47.6)$ \\
Do not collect data from older populations (age $\geq 65$ years) & \\
Unclear description of anatomical site by condition (when applicable) & $\mathrm{d}$ \\
OA & $51(98.0)$ \\
Low back pain/neck pain & $9(19.1)$ \\
\hline
\end{tabular}

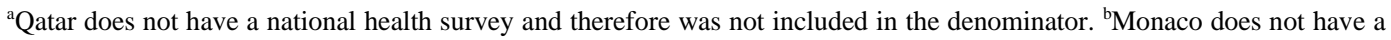
national health survey and therefore was not included in the denominator. ${ }^{\mathrm{c}}$ The Federated States of Micronesia do not have a national health survey and therefore were not included in the denominator. ${ }^{\mathrm{d}}$ Only one survey specifically described the site of OA and measured the prevalence of knee and hip OA separately. Surveys from nine countries used unclear terminology to refer to either low back pain or neck pain (e.g. 'back pain'): Australia, Brazil, Costa Rica, Djibouti, Grenada, Liechtenstein, USA, Uruguay and the Republic of Yemen.

\section{Proportion of national health surveys that measured the prevalence of musculoskeletal conditions}

Sixty-two (36.4\%) national health surveys measured the prevalence of at least one musculoskeletal condition (Fig. 1). Most were from high-income countries [43 (69.3\%)] and the Europe and Central Asia region [32 (51.6\%)]. The most commonly measured prevalence was for OA [53 $(85.5 \%)]$, followed by low back pain [39 $(62.9 \%)]$ and neck pain [37 $(59.7 \%)]$. 
FIG. 1 Proportion of national health surveys

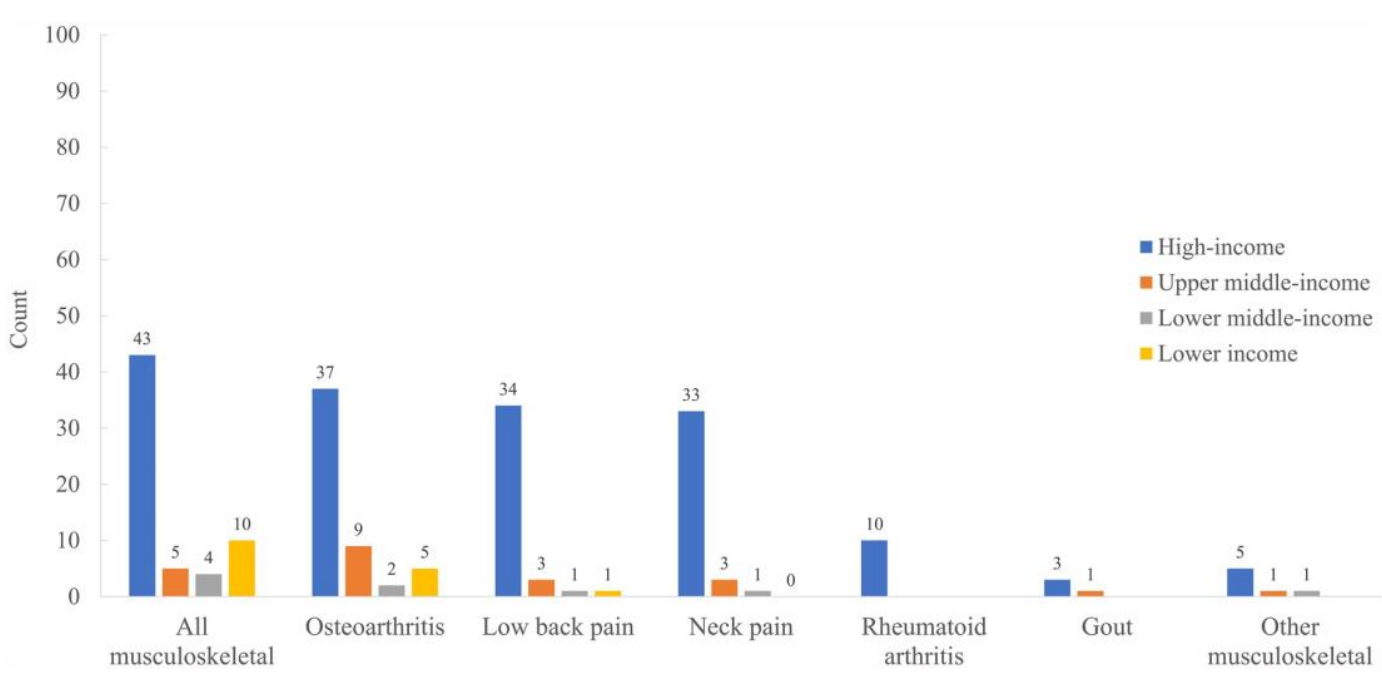

Proportion of national health surveys that contained at least one question on the prevalence of any musculoskeletal condition, OA, low back pain, neck pain, RA, gout and other musculoskeletal conditions. Data are disaggregated by income level.

A very small number of surveys measured the prevalence of RA [10 (16.4\%)] and gout [3 (4.9\%)]. In two surveys (USA and Canada), the prevalence of RA and gout were asked about in the same question.

Proportion of national health surveys that measured condition-specific assessment of activity limitation, severity of pain and work absence

Only a small proportion of surveys assessed condition-specific activity limitation [6 (9.8\%)], pain severity $[5(8.2 \%)]$ and work absence $[1(1.6 \%)]$ and they were all related to either low back pain, neck pain or OA.

\section{National health surveys that followed the standardized definitions of low back pain}

Only one country (Solomon Islands) used a definition of low back pain (the minimal definition) recommended by Dionne et al. [12].

\section{Discussion}

Only $36.4 \%$ of national health surveys collected data about the prevalence of musculoskeletal conditions. Most prevalence data were from high-income countries (68.9\%). Only a very small proportion (0.6-3.6\%) of national health surveys assessed the consequences of musculoskeletal conditions in terms of condition-specific activity limitation, pain severity or disease-related work absence. These data were collected for low back pain and/or OA but not for any other musculoskeletal condition. 
Our study has several strengths. We employed a thorough search strategy to maximize the inclusion of national health surveys, which included online searching on each country's National Statistics Office's website. A limitation of our study was that we were unable to retrieve 44 surveys $(20.0 \%)$. An additional limitation of the study is the inclusion of 28 surveys released $>10$ years ago. However, we used comprehensive methods to try to obtain all the surveys and surveys released $>10$ years ago accounted for only $16.4 \%$ of the data. Another potential limitation was that we screened the first 20 records on Google. This was a pragmatic decision, given that we searched on Google for each one of the 218 countries. However, we supplemented our searches with other methods to minimize the risk of missing relevant documents.

National health surveys are considered the main data source to support evidence-based policy planning, development and monitoring globally [10-12]. However, we demonstrate that monitoring the disease burden of musculoskeletal conditions in these reports has been poor. Capturing information about musculoskeletal conditions in national health surveys, particularly in LMICs, should be a priority, given the lack of high-quality observational studies [6] and the fact that burden of disease statistics in many of these countries are often based on estimations [13-15]. Some initiatives to collect prevalence data on musculoskeletal conditions, such as the Community Oriented Program for Control of Rheumatic Diseases (COPCORD), have contributed to improving data availability on musculoskeletal conditions in LMICs [16]. However, very few COPCORD initiatives have continued beyond the initial survey. This limits the understanding of changes in the prevalence of those conditions over time [17].

Efforts to standardize national health surveys across countries have been made over the last few decades. For example, the statistical office of the European Union (Eurostat) has recently rolled out the third wave of the European Health Interview Survey (EHIS) across countries that are members of the European Union. All 15 third-wave EHISs we included in our study measured the prevalence of low back pain, neck pain and OA in a very similar fashion. However, only Estonia's national health survey measured activity limitation due to low back pain, neck pain and OA, but not due to other musculoskeletal conditions.

Another example of a coordinated effort towards standardization of surveys across countries is the Demographic and Health Surveys (DHS) from the US Agency for International Development (USAID). The DHS programme has completed surveys for $>80$ and LMICs since its inception in 1984 and these have been used to inform policy in those countries $[10,18]$. Only 6 of 68 DHS we included $(8.8 \%)$ contained questions to quantify the prevalence of musculoskeletal conditions. Furthermore, most of the DHS do not interview people $\geq 65$ years of age, therefore prevalence estimates for these conditions will be underestimated. DHS usually focus on topics related to the health of women of child-bearing age, which likely explains the exclusion of older people. The exclusion of older people and the absence of questions regarding musculoskeletal conditions in most DHS represent an important opportunity loss for many countries.

The fact that only one country (Solomon Islands) has adopted a standardized definition of low back pain published more than a decade ago demonstrates the large scope for improvement in the monitoring of musculoskeletal conditions surveillance in national health surveys worldwide [19]. It also highlights the need for translating research findings in this area into practice. These challenges are particularly important in LMICs.

A potential barrier to the integration of questions covering musculoskeletal conditions in national health surveys is a lack of recognition of musculoskeletal health as a health priority. For example, despite ample evidence that musculoskeletal conditions are a major contributor to the disability burden of non-communicable diseases worldwide, non-communicable disease policies are mostly silent on them [20]. 


\section{Conclusion}

The burden of musculoskeletal conditions is increasing and countries need to respond appropriately to the challenges imposed by them. National health surveys are used to guide policy planning, but we have shown that most of them currently neglect musculoskeletal conditions.

\section{Acknowledgements}

G.F., J.Z., M.O.K. and J.O. conceived the idea and were responsible for the study design, literature search, data collection, data analysis, data interpretation and writing. R.B. was responsible for the study design, data interpretation and writing. P.K. was responsible for the literature search, data collection, data analysis, data interpretation and writing. L.C. was responsible for data collection, data analysis, data interpretation and writing. C.M. conceived the idea was responsible for the study design, data interpretation and writing.

Funding: This study received no specific funding. R.B. is supported by a Senior Principal Research Fellowship from Australia's National Health and Medical Research Council (NHMRC). C.M. is supported by a Principal Research Fellowship from the NHMRC. J.Z. is supported by an NHMRC fellowship. The corresponding author (G.F.) had full access to all the data in the study and had final responsibility for the decision to submit for publication.

Disclosure statement: The authors have nothing to declare.

\section{Data availability statement}

Data will be made available upon request.

\section{References}

1. Kyu HH, Abate D, Abate KH et al. Global, regional, and national disability-adjusted life-years (DALYs) for 359 diseases and injuries and healthy life expectancy (HALE) for 195 countries and territories, 1990-2017: a systematic analysis for the Global Burden of Disease Study 2017. Lancet 2018;392:1859-922.

2. Hartvigsen J, Hancock MJ, Kongsted A et al. What low back pain is and why we need to pay attention. Lancet 2018;391:2356-67.

3. Dieleman JL, Cao J, Chapin A et al. US health care spending by payer and health condition, 19962016. JAMA 2020;323:863-84.

4. Safiri S, Kolahi AA, Hoy D et al. Global, regional and national burden of rheumatoid arthritis 19902017: a systematic analysis of the Global Burden of Disease Study 2017. Ann Rheum Dis 2019;78:1463-71.

5. Blyth FM, Briggs AM, Schneider CH, Hoy DG, March LM. The global burden of musculoskeletal pain-where to from here? Am J Public Health 2019;109:35-40.

6. Hoy D, Bain C, Williams G et al. A systematic review of the global prevalence of low back pain. Arthritis Rheum 2012;64:2028-37.

7. Pereira D, Peleteiro B, Araújo J et al. The effect of osteoarthritis definition on prevalence and incidence estimates: a systematic review. Osteoarthritis Cartilage 2011;19:1270-85.

8. Dionne CE, Dunn KM, Croft PR et al. A consensus approach toward the standardization of back pain definitions for use in prevalence studies. Spine (Phila Pa 1976) 2008;33:95-103.

9. Tolonen H, Koponen P, Al-kerwi A et al. European health examination surveys - a tool for collecting objective information about the health of the population. Arch Public Health 2018;76:38.

10. Corsi DJ, Neuman M, Finlay JE, Subramanian SV. Demographic and health surveys: a profile. Int J Epidemiol 2012;41:1602-13.

11. Oyebode O, Mindell JS. A review of the use of health examination data from the Health Survey for England in government policy development and implementation. Arch Public Health 2014;72:24. 
12. Weisz G. Epidemiology and health care reform: the National Health Survey of 1935-1936. Am J Public Health 2011;101:438-47.

13. Safiri S, Kolahi A-A, Hoy D et al. Global, regional, and national burden of neck pain in the general population, 1990-2017: systematic analysis of the Global Burden of Disease Study 2017. BMJ 2020;368:m791.

14. Cross M, Smith E, Hoy D et al. The global burden of hip and knee osteoarthritis: estimates from the Global Burden of Disease 2010 study. Ann Rheum Dis 2014;73:1323-30.

15. Wu A, March L, Zheng X et al. Global low back pain prevalence and years lived with disability from 1990 to 2017: estimates from the Global Burden of Disease Study 2017. Ann Transl Med 2020;8:299.

16. Chopra A. COPCORD - an unrecognized fountainhead of community rheumatology in developing countries. J Rheumatol 2004;31:2320-2.

17. Chopra A. The COPCORD world of musculoskeletal pain and arthritis. Rheumatology 2013;52:19251928.

18. Short Fabic M, Choi Y, Bird S. A systematic review of Demographic and Health Surveys: data availability and utilization for research. Bull World Health Org 2012;90:604-12.

19. Hoy DG, Raikoti T, Smith E et al. Use of the Global Alliance for Musculoskeletal Health survey module for estimating the population prevalence of musculoskeletal pain: findings from the Solomon Islands. BMC Musculoskelet Disord 2018;19:292.

20. Briggs AM, Persaud JG, Deverell ML et al. Integrated prevention and management of noncommunicable diseases, including musculoskeletal health: a systematic policy analysis among OECD countries. BMJ Global Health 2019;4:e001806. 